\title{
Management of post-operative complications in open ventral hernia repair
}

\author{
Sean C. O'Connor, Alfredo M. Carbonell \\ Department of Surgery, Prisma Health-Upstate, University of South Carolina School of Medicine-Greenville, Greenville, SC 29605, USA.
}

Correspondence to: Dr. Alfredo M. Carbonell, Department of Surgery, Prisma Health-Upstate, University of South Carolina School of Medicine-Greenville, 3rd Floor Support Tower, 701 Grove Rd, Greenville, SC 29605, USA. E-mail: alfredo.carbonell@prismahealth.org

How to cite this article: O'Connor SC, Carbonell AM. Management of post-operative complications in open ventral hernia repair. Plast Aesthet Res 2019;6:26. http://dx.doi.org/10.20517/2347-9264.2019.38

Received: 10 Oct 2019 First Decision: 6 Nov 2019 Revised: 13 Nov 2019 Accepted: 15 Nov 2019 Published: 29 Nov 2019

Science Editor: Alexander F. Mericli Copy Editor: Jing-Wen Zhang Production Editor: Tian Zhang

\begin{abstract}
Hernia repair is the most common general surgical procedure performed in the United States; however, historically, there has been a surprising lack of consensus regarding hernia complications and their management. The development of international, prospectively-collected databases such as the Americas Hernia Society Quality Collaborative has introduced a new era of evidence-based practice around the prevention and management of these complications. This review seeks to equip surgeons with evidence-based techniques for prevention and management of the most common complications of open ventral hernia repair.
\end{abstract}

Keywords: Hernia repair, complications, surgical site infection, seroma, flap necrosis, mesh exposure, interparietal hernia, recurrent hernia, enterocutaneous fistula

\section{INTRODUCTION}

Complications after hernia repair are divided into general surgical complications and hernia specific complications. The Clavien Dindo classification system can be used to classify general surgical complications based on severity and required interventions ${ }^{[1]}$. The Ventral Hernia Working Group describes hernia specific complications as surgical site occurrence (SSO) to standardize the nomenclature when studying outcomes after hernia repair. SSO is a category of complications that includes surgical site infection (SSI), seroma, hematoma, wound dehiscence, and enterocutaneous fistula ${ }^{[2]}$. Petro et al. ${ }^{\left[{ }^{3}\right]}$ found an association between SSO and the complexity of hernia repair using a hernia staging system that focuses 
on the defect size and presence of contamination. Stage 1 hernias are less than $10 \mathrm{~cm}$ and in clean fields and have been found to have a SSO and recurrence rate of $10 \%$. Stage 2 hernias are larger, $10-20 \mathrm{~cm}$ or $<10 \mathrm{~cm}$ in contaminated fields, and have an SSO rate of about $20 \%$ and recurrence rate of $15 \%$. Stage 3 hernias are $>20 \mathrm{~cm}$ wide or $>10 \mathrm{~cm}$ in contaminated fields and have the highest risk of SSO at $42 \%$ and recurrence of $26 \%$. This system allows surgeons to have a frank discussion with their patients pre-operatively about their expected complication risk.

While such staging systems are helpful in risk stratification, SSO remains a broad category including 5 types of complications and fails to account for their clinical significance. Separate systems exist for each category of SSO to document a difference between small asymptomatic seromas and large, symptomatic seromas requiring intervention for example ${ }^{[4]}$.

\section{EARLY COMPLICATIONS}

\section{Mesh infection}

Much attention has been paid to prevention of SSI in hernia repair, especially with the increased use of prosthetic mesh. These are divided into superficial, deep, and organ space SSI and are directly correlated with the level of contamination during the case. Superficial SSI should be managed using general surgical principles of drainage and, possibly, short-course antibiotics. Management of deep and organ space SSI hinges greatly on the concern for infection of the prosthetic mesh ${ }^{[5]}$.

The true diagnosis of mesh infection requires a positive culture of fluid from around the mesh or a culture of the mesh itself. Periprosthetic fluid on imaging can often represent sterile seroma or hematoma, so fluid should be aspirated and sent for culture if there is concern for mesh infection. However, deep infections should be treated aggressively, and with a high index of suspicion, as seeding of the mesh can be subclinical and may lead to long-term complications. A retrospective review of 21 mesh infections found that $76 \%$ of mesh infections were due to Staphylococcus aureus and about half of these were methicillin-resistant. The minority of infections are due to Staphylococcus epidermidis or Streptococcus pyogenes or Gram-negative species of Escherichia coli or Klebsiella in the case of gastrointestinal contamination ${ }^{[6,7]}$.

Prevention of mesh infection begins with tailoring the type of mesh chosen for the clinical scenario. We recommend the use of medium-weight macroporous polypropylene (PP) in clean fields due to their resistance to biofilm formation, improved clearance of infection, and repair durability compared to PTFEbased meshes ${ }^{[5]}$. In contaminated or clean-contaminated fields, the evidence on mesh selection is not as clear, and absorbable mesh is recommended due to the high risk of synthetic mesh infection. Itani et al. ${ }^{[8]}$ and Rosen et al. ${ }^{[9]}$ showed in a prospective trial that using biosynthetic absorbable mesh resulted in improved SSI rates $(18 \%$ vs. $35 \%)$ as well as long-term recurrence rates (17\% vs. $28 \%)$ when compared to previous trials of porcine mesh in these settings More recently, however, macroporous synthetic mesh has been found to have equivalent infection rates in contaminated fields and is also an acceptable option ${ }^{[10]}$.

Once a mesh infection has been confirmed, early source control and antibiotics are required. An effort should be made to salvage the mesh, avoiding removal. Patients showing signs of sepsis may require early operative intervention characterized by pulse-lavage antibiotic solution irrigation, followed by wide closed suction drain placement adjacent to the mesh and fascial closure once again if the mesh had been placed retromuscular. In the setting of florid sepsis, source control, wound packing, and interval abdominal wall closure is often all the patient will tolerate. Some small series have shown the success of mesh removal with single stage primary closure with bilateral myofascial rectus abdominis release; however, the hernia recurrence rates range $35 \%-88 \%{ }^{[11,12]}$. This setting is an ideal application for absorbable biosynthetic mesh, where it can substantially reduce recurrence rates down to $17 \%^{\left[{ }^{[}\right]}$. Absorbable mesh should be placed as a sublay in the retrorectus space and can be placed at the index operation or in a staged approach. 
In patients without systemic signs of sepsis, an effort toward mesh salvage should be attempted. Patients should be immediately started on broad spectrum antibiotics and these can be narrowed based on culture data. Percutaneous drainage of any fluid surrounding the mesh should be performed and the drain should be left in place until source control is satisfactory. Any overlying necrotic tissue should be surgically debrided; exposed mesh can be irrigated with antibiotics; and negative pressure wound management systems can be placed to allow granulation formation over the mesh. Success rates of mesh salvage protocols have been promising; however, this is very dependent on the structure of the mesh, with macroporous mesh in the retrorectus position having the highest salvage rate ${ }^{[13]}$. PTFE, multifilament polyester, and heavy weight, microporous PPE, however, almost universally require explantation once infected due to their poor tissue ingrowth and biofilm formation that prevent clearance of bacteria ${ }^{[14]}$. Thus, upfront excision of these mesh types is recommended to expedite recovery and ultimate reconstruction ${ }^{[12]}$.

\section{Seroma}

Every patient who has an open hernia repair will develop some element of a seroma due to the potential spaces created in the abdominal wall. The vast majority of seromas are asymptomatic and most resolve without any intervention. Morales-Conde et al. ${ }^{[4]}$ created a seroma classification system, in which they defined seromas as a complication only if they are symptomatic, persist longer than 6 months, or become infected (Types III and IV). Clinically detected seromas that last less than 6 months (Types I and II) are classified as an incidental finding, reflecting that these are considered normal sequela of the operation. Type III seromas last longer than 6 months or become symptomatic, but do not require intervention, while Type IV seromas are symptomatic and require intervention. In this classification system, only Type III and IV seromas should be considered true complications as they affect the clinical progression of the patient.

Routine drain placement to prevent seromas has been debated over concerns that drains in direct contact with the mesh could increase the risk of infection. In 2017, Krpata et al. ${ }^{[15]}$ queried the Americas Hernia Society Quality Collaborative (AHSQC) and reviewed 200 cases where drains were placed in the retromuscular space and 100 cases where they were not. These cases were matched based upon hernia complexity and comorbidities. They found no difference in superficial, deep, or organ space SSI between the groups, but there was an overall increase in SSO in the no drain group (20\%vs. 10\%, $P=0.02)$ with seroma formation being the most significant increase ( $8 \% v$ s. $2 \%, P=0.01)$. They did not, however, see a difference in surgical site occurrences requiring procedural intervention (SSOPI), suggesting that most of these seromas resolved without intervention. They concluded that routine drain placement does not increase the risk for infection and may reduce the risk of seroma formation, although the clinical significance of seroma formation continues to be debated.

Once a seroma has grown to cause pain, impede normal activities, or persist for longer than 6 months, intervention is recommended for the alleviation of symptoms. The primary treatment option is percutaneous drainage, which can be done with serial aspirations or by leaving a secured catheter in place and monitoring the output overtime. These can either be performed in the office blindly or under radiographic guidance with ultrasound or computed tomography, as long as a sterile technique is followed to prevent secondary infection of the seroma. For seromas refractory to simple drainage, chemical or mechanical sclerotherapy have both been described. Chemical sclerosing agents including talc, tetracycline, doxycycline, ethanol, erythromycin, fibrin glue, and povidone/iodine have all been used with high success rates and few complications; however, only small case series exist in the literature ${ }^{[16,17]}$. Lehr and Schuricht ${ }^{[18]}$ described endoscopic ablation of the inner lining of the seroma using the argon beam in extreme cases. Three ports are inserted into the seroma and the fluid is drained, followed by insufflation to allow endoscopic guidance of the argon beam to ablate the entire lining of the seroma. These advanced techniques should be used only in extreme cases, after failure of watchful waiting and percutaneous drainage. 


\section{Flap necrosis}

Any surgeon performing complex ventral hernia repair should have an intimate knowledge of the blood supply to the abdominal wall. The inferior epigastric artery supplies the majority of blood flow to the rectus abdominis muscle with collaterals to the superior epigastric from the internal mammary. These send perforating branches anteriorly through the anterior rectus sheath to supply the subcutaneous fat and overlying abdominal wall skin. The largest concentration of these perforators lies within $10 \mathrm{~cm}$ of the umbilicus in all directions and preservation of this pedicle is paramount in preventing tissue ischemia and subsequent SSO. This principle has driven innovation in abdominal wall reconstruction to preserve as much of the abdominal wall blood supply as possible ${ }^{[19]}$.

To minimize large subcutaneous flaps that contribute to skin necrosis, retrorectus placement of the mesh whenever possible in a "sublay" technique has been widely popularized in recent years ${ }^{[20]}$. If a component separation is required for closure of the midline, the debate over wound complication rates between anterior component separation (ACS) and transversus abdominis release (TAR) still continues. The TAR repair has been described extensively with very low wound complication rate. In Novitsky's 2012 paper, only one of the 42 original patients developed skin flap necrosis with the TAR technique, and this patient had subcutaneous flaps raised during the initial operation ${ }^{[21]}$. Harth et al. ${ }^{[22]}$ presented similar findings when studying the flaps raised in panniculectomy during hernia repair. They found a $70 \%$ wound complication rate in the panniculectomy group with $40 \%$ requiring return to the operating room for debridement. This highlights the morbidity associated with large subcutaneous flaps on the abdominal wall when combined with hernia repair.

For large hernias in which mobility of the anterior elements prevents closure of the linea alba, an ACS may be required to gain mobility of greater than $15 \mathrm{~cm}$. In this case, a periumbilical perforator sparing technique preserves blood supply to the skin flap and has been shown to reduce SSO and skin necrosis ${ }^{[2,24]}$. This technique involves tunneling around the periumbilical pedicle of perforator vessels to gain access to the external oblique muscle release, and can be done either open or endoscopically ${ }^{[19]}$. If concerns of ischemia remain, the flap can be evaluated with fluorescence angiography to thoroughly evaluate the viability of skin and subcutaneous tissue ${ }^{[25]}$. The mesh should still be placed in the retrorectus space to prevent further undermining of the flap, and to provide another barrier between the skin and the mesh should skin necrosis or SSI occur ${ }^{[20]}$.

\section{Interparietal hernia}

Interparietal hernias occur in retromuscular repairs due to dehiscence of the posterior rectus fascia/ peritoneal closure, allowing intrabdominal contents to herniate between the posterior fascia and the mesh. Bowel can become acutely incarcerated in this layer, or adhesions and fistulas can form due to direct exposure of the mesh to the bowel. This is a rare complication with only small case series in existence, the largest of which describes 9 (1.8\%) cases out of 511 retromuscular hernia repairs. In this series, the majority of patients presented with acute obstruction within 30 days of the index operation. One patient presented over 10 months after surgery with resolving abdominal pain and 2 were found incidentally on imaging for other reasons. Because of its rarity, the diagnosis can be difficult and is often missed because of the atypical appearance on CT scan. On cross sectional imaging, the posterior sheath can appear as a shelf with fluid, omentum, or bowel lying between it and the overlying rectus muscles [Figure 1$]^{[26,27]}$.

Lack of bowel function within a few days of surgery should be treated with a high index of suspicion and be evaluated with a CT scan to look for acute causes of bowel obstruction. In the case of interparietal hernia, management then depends on the timing of presentation. In the acute period, the mesh can be in direct contact with bowel increasing the risk of adhesions. The posterior defect should be bridged with a piece of absorbable synthetic or biologic mesh; this acts as a barrier between bowel and the mesh to allow 

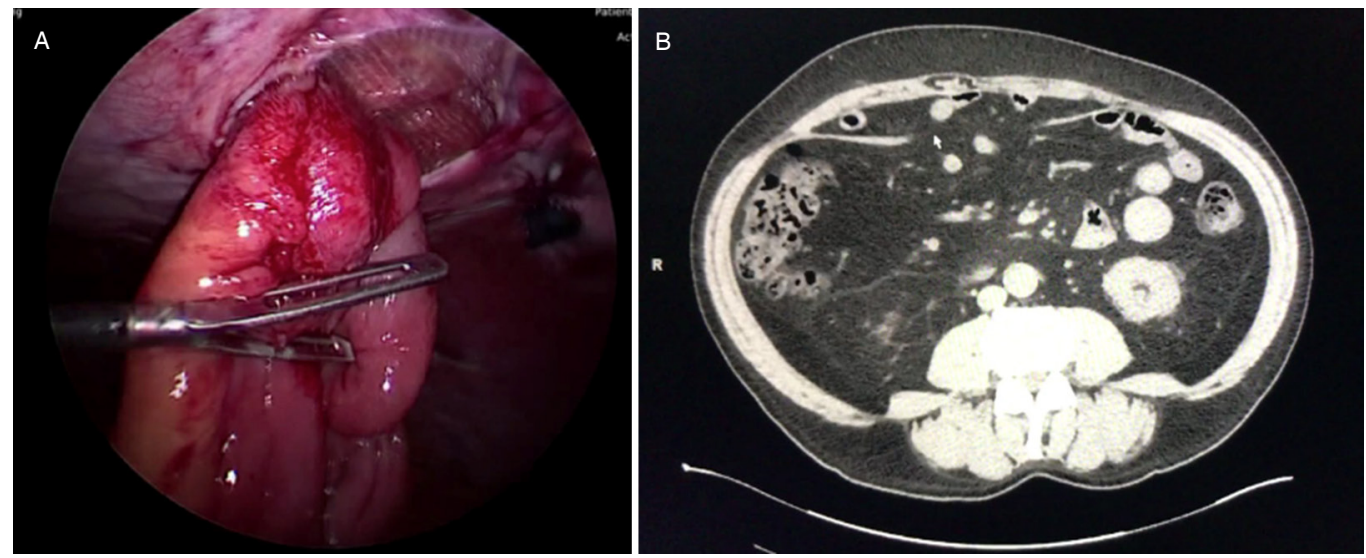

Figure 1. A: laparoscopic image of bowel herniated above the posterior rectus sheath and in contact with the exposed mesh. B: CT image showing the dehisced posterior rectus sheath with bowel herniated above. Linea alba is intact with no full thickness herniation

time for reperitonealization. In the case of late presentations, 2-3 weeks post operatively, the mesh should be completely peritonealized, and the objective here is to obliterate the space above the posterior sheath to prevent bowel from herniating above. This can be done by simply lifting the edges of the posterior sheath with either transfascial sutures or an absorbable tacking device to attach it to the mesh and obliterate the potential space ${ }^{[27]}$.

\section{LATE COMPLICATIONS}

\section{Mesh exposure}

A long-term complication of SSI or flap necrosis can be exposure of the mesh. One of the arguments for retrorectus placement of mesh is that the rectus acts as another layer between the mesh and the skin, preventing exposure of the mesh if the skin and subcutaneous tissue are debrided. However, when mesh does become exposed, it can create a chronic wound that is difficult to epithelialize. A trial of mesh salvage is almost always appropriate when the patient is stable and not septic. Once necrotic tissue has been fully debrided, negative pressure wound therapy can be used to encourage granulation formation through the mesh pores, ultimately covering the mesh ${ }^{[28]}$. In animal models, the degree and speed of tissue integration is greatest with macroporous $(1.8 \mathrm{~mm} \times 3.4 \mathrm{~mm}$ pore size $)$ compared to microporous mesh $(0.9 \mathrm{~mm} \times 1 \mathrm{~mm}$ pore size $)^{[29]}$. This is thought to be one of the contributing factors in the improved salvage rate of macroporous mesh $^{[12]}$. Once a thick base of granulation tissue covers the entire mesh, the wound can be covered using a split-thickness skin graft.

Berrevoet et al. ${ }^{[13]}$ reviewed 54 cases of macroporous mesh placed in the retrorectus space that was exposed or infected. Mesh salvage was achieved in all patients studied, with a mean wound closure time of 44 days (range, 26-73 days) and 5 dressing changes. If chronic wounds or draining sinus tracts exist, small portions of the exposed mesh can be debrided to improve wound healing. Patients should be supported with enteral or parenteral nutrition to avoid protein calorie malnutrition, and immunosuppressants should be minimized to improve wound healing. PTFE, multifilament polyester, and heavy weight, microporous PPE are more difficult to salvage due to their poor tissue ingrowth and biofilm formation, which prevents complete clearance of bacteria. If explantation is required, meticulous removal of all mesh and synthetic material, including suture and tacks, should be performed. Primary closure with staged repeat repair should be performed if possible, or use of biosynthetic mesh if required for coverage of intrabdominal contents ${ }^{[9]}$.

\section{Enterocutaneous fistula}

Enterocutaneous fistula (ECF) is a feared long-term complication of hernia repair, occurring on average 2 years after the index operation. These often present as a chronic mesh infection that is followed by bilious 
or feculent drainage and require multiple interventions and hospital admissions to resolve. The literature suggests that avoidance of enterotomies at the index operation is the best way to prevent fistula formation post operatively. In a large database review of elective ventral hernia repairs, enterotomy or unplanned bowel resection $(\mathrm{EBR})$ increased the ECF formation rate from $0.7 \%$ to $7.1 \%(P<0.01)$. The authors found that repair of recurrent hernias where mesh had been placed at the index operation had a significantly higher rate of EBR compared to primary hernia repair or recurrent repair after prior suture repair alone (20.4\% vs. 5.7\%, $P<0.001)$. This highlights the difficulty of reoperative hernia surgery and the importance of meticulous lysis of adhesions to prevent devastating complications in the future ${ }^{[7,30]}$.

For many years, it has been thought that PP mesh would have a higher risk of ECF formation due to the vigorous foreign body response and inflammation it incites when compared to polyester or PTFE. However, long-term data on retrorectus technique have shown no increased ECF formation with PP mesh when compared to $\mathrm{PTFE}^{[31]}$. This was further supported by Brandi et al ${ }^{[32]}$, who reported no ECF even with uncoated polypropylene mesh in the intraperitoneal position.

Management of ECF should start with conservative measures and control of contamination, because immediate mesh explanation is associated with a high risk of EBR, which could result in even further fistula formation $^{[7]}$. Macroporous polypropylene and polyester mesh are more likely to be salvaged than PTFE due to its poor tissue ingrowth and biofilm formation. Conservative management should include opening the tract to control sepsis, nutritional support with TPN, somatostatin, downstream decompression, and appropriate wound care, which can lead to spontaneous closure in many cases ${ }^{[33]}$. If the fistula fails to close, it is recommended to wait for 6 month to allow for spontaneous closure and maturing of adhesions before mesh excision and bowel resection is undertaken ${ }^{[34]}$. In a recent review of the AHSQC database, Kao et al. ${ }^{[35]}$ looked at outcomes from partial mesh excision (PME) vs. complete mesh excision in clean, clean-contaminated, contaminated, and dirty wounds as well as cases with ECF. Not surprisingly, they found a higher rate of SSI, SSO, SSOPI, and reoperation in the PME group in cases of ECF, contaminated or dirty wounds ${ }^{[35]}$. In the case of mesh infection or fistula, all permanent pieces of mesh and suture should be removed as they will serves as a nidus for future infection or wound complications.

\section{Hernia recurrence}

It is well established at this point that placement of synthetic mesh during hernia repair reduces hernia recurrence rates compared to suture repair alone. The largest study on recurrence was on the Denmark national health system data bank, which followed 3242 patients for a median of 5 years and found a $12.3 \%$ recurrence rate with mesh compared to $17.1 \%$ without ${ }^{[36]}$. The most common site of recurrence is in the midline directly through central fractures in the mesh, accounting for up to $39.6 \%$ of recurrences ${ }^{[37]}$. Factors that increase the risk of mesh fracture can be both technical in nature and due to material weakness. There has been a shift in mesh material towards lightweight mesh due to its improved flexibility and decreased shrinkage over time as there is less inflammatory reaction to a decreased volume of material ${ }^{[38]}$. However, this comes at the price of decreasing its overall strength, especially when combined with a macroporous configuration to allow for bacterial clearance. Petro et al. ${ }^{[39]}$ recently published their experience with macroporous lightweight polyester placed in the retrorectus position on 36 patients. Of the eight (22\%) recurrences after 13 months, seven (19\%) were found to have a central mesh fracture as the mechanism of recurrence. Warren et al. ${ }^{[37]}$ also found that the use of lightweight polypropylene mesh was an independent risk factor for central mesh fracture. Because of this, medium- or heavyweight mesh is recommended, especially when using a macroporous mesh.

While the material of the mesh itself may be partially to blame, there are also technical factors that place patients at risk for central mesh fracture. Failure to close the midline or midline dehiscence leaves the mesh unsupported by the abdominal wall, causing increased stress and eventual fracture of the mesh. There is 
no substitute for good technique when closing the linea alba, utilizing small bites and adequate myofascial release to bring the midline together with minimal tension ${ }^{[40]}$. Mesh fracture has also been associated with SSI, which weakens the fascial layers and in turn places mechanical stress on the mesh. Mesh fixation with sutures, staples, and tacks has not been associated with fracture, although this was previously thought to be a possible source. Failures around the mesh are most commonly found at the site of transfascial fixation sutures, which pull through and weaken the abdominal wall, or due to inadequate mesh-tissue overlap in all directions.

Maloney et al ${ }^{[24]}$ reviewed a large hernia database to determine risk factors for recurrence after component separations. There was a higher risk of SSO with ACS leading to a higher overall recurrence rate; however, on univariate analysis, there was no difference between anterior and posterior component separations, and no association with smoking status, steroid use, diabetes, or peripheral vascular disease. There was, however, an association with BMI greater than 35, use of absorbable mesh, SSO, SSI, and failure to close the fascia. The exact effect of BMI on recurrence after hernia repair is difficult to ascertain due to heterogeneity in the literature, although an increase in hernia recurrence has consistently been found with BMI $>30-35^{[41]}$. Interestingly, recurrence was not associated with the size of the defect as long as the fascia was closed, reinforcing the importance of adequate myofascial release and midline closure technique.

Unfortunately, recurrent hernia itself is a risk factor for recurrence of subsequent repairs, meaning that many patients enter a vicious cycle of multiple failed repairs. The risks of complete mesh excision can be significant, often requiring extensive lysis of adhesions with risk of bowel injury. In addition, if the mesh is well incorporated, there is a risk of destruction of the native abdominal wall components, making subsequent repairs more difficult. In the aforementioned review of the AHSQC database, Kao et al. ${ }^{[35]}$ also compared partial $v s$. complete mesh excision in clean cases such as excision for pain or recurrent hernia. In these cases, there was no difference in SSO, SSI, SSOPI, or reoperation in patients who only underwent partial excision of the mesh. This suggests that, in the case of small recurrence without any infection, it is reasonable to repair the hernia without complete excision of previous mesh. If the recurrent defect is through the mesh, primary repair should be performed with permanent suture bites through the previous mesh to reestablish its continuity. Defects above or lateral to the mesh can be repaired with underlay or sublay technique either laparoscopically or open. Mesh fractures larger than $2 \mathrm{~cm}$ should be treated with explantation and repeat repair with permanent mesh to prevent layering of mesh in the abdominal wall.

\section{DECLARATIONS}

\section{Authors' contributions}

Made substantial contributions to conception and design of the study and performed data analysis and interpretation: O'Connor SC, Carbonell AM

\section{Availability of data and materials}

Not applicable.

\section{Financial support and sponsorship}

None.

\section{Conflicts of interest}

Sean C. O'Connor, M.D. has no disclosures. Alfredo M. Carbonell, D.O. has received honoraria from W.L. Gore and Associates, Ethicon Inc. and Intuitive.

\section{Ethical approval and consent to participate}

Not applicable. 


\section{Consent for publication}

Not applicable.

\section{Copyright}

(c) The Author(s) 2019.

\section{REFERENCES}

1. Dindo D, Demartines N, Clavien PA. Classification of surgical complications: a new proposal with evaluation in a cohort of 6336 patients and results of a survey. Ann Surg 2004;240:205-13.

2. Kanters AE, Krpata DM, Blatnik JA, Novitsky YM, Rosen MJ. Modified hernia grading scale to stratify surgical site occurrence after open ventral hernia repairs. J Am Coll Surg 2012;215:787-93.

3. Petro CC, O’Rourke CP, Posielski NM, Criss CN3, Raigani S, et al. Designing a ventral hernia staging system. Hernia 2016;20:111-7.

4. Morales-Conde S. A new classification for seroma after laparoscopic ventral hernia repair. Hernia 2012;16:261-7.

5. Cevasco M, Itani KMF. Ventral hernia repair with synthetic, composite, and biologic mesh: characteristics, indications, and infection profile. Surg Infect (Larchmt) 2012;13:209-15.

6. Cobb WS, Carbonell AM, Kalbaugh CL, Jones Y, Lokey JS. Infection risk of open placement of intraperitoneal composite mesh. Am Surg 2009;75:762-7.

7. Sanchez VM, Abi-Haidar YE, Itani KMF. Mesh infection in ventral incisional hernia repair: incidence, contributing factors, and treatment. Surg Infect (Larchmt) 2011;12:205-10.

8. Itani KMF, Rosen M, Vargo D, Awad SS, Denoto G, et al. Prospective study of single-stage repair of contaminated hernias using a biologic porcine tissue matrix: The RICH Study. Surgery 2012;152:498-505.

9. Rosen MJ, Bauer JJ, Harmaty M, Carbonell AM, Cobb WS, et al. Multicenter, prospective, longitudinal study of the recurrence, surgical site infection, and quality of life after contaminated ventral hernia repair using biosynthetic absorbable mesh: The COBRA Study. Ann Surg 2017;265:205-11.

10. Carbonell AM, Criss CN, Cobb WS, Novitsky YW, Rosen MJ. Outcomes of synthetic mesh in contaminated ventral hernia repairs. J Am Coll Surg 2013;217:991-8.

11. Szczerba SR, Dumanian GA. Definitive surgical treatment of infected or exposed ventral hernia mesh. Ann Surg 2003;237:437-41.

12. Kao AM, Arnold MR, Augenstein VA, Heniford BT. Prevention and treatment strategies for mesh infection in abdominal wall reconstruction. Plast Reconstr Surg 2018;142:149-55S.

13. Berrevoet F, Vanlander A, Sainz-Barriga M, Rogiers X, Troisi R. Infected large pore meshes may be salvaged by topical negative pressure therapy. Hernia 2013;17:67-73.

14. Hawn MT, Gray SH, Snyder CW, Graham LA, Finan KR, et al. Predictors of mesh explantation after incisional hernia repair. Am J Surg 2011;202:28-33.

15. Krpata DM, Prabhu AS, Carbonell AM, Haskins IN, Phillips S, et al. Drain placement does not increase infectious complications after retromuscular ventral hernia repair with synthetic mesh: an AHSQC analysis. J Gastrointest Surg 2017;21:2083-9.

16. Sood A, Kotamarti VS, Therattil PJ, Lee ES. Sclerotherapy for the management of seromas: a systematic review. Eplasty 2017;17:e25.

17. Al Daoud F, Thayer A, Sachwani Daswani G, Maraqa T, Perinjelil V, et al. Management of chronic abdominal wall seroma with Doxycycline sclerotherapy using a Negative Pressure Wound Therapy System KCI-V.A.C.UltaTM - A case report. Int J Surg Case Rep 2018;51:25-8

18. Lehr SC, Schuricht AL. A minimally invasive approach for treating postoperative seromas after incisional hernia repair. J Soc Laparoendosc Surg 2001;5:267-71.

19. Rosen MJ. Atlas of abdominal wall reconstruction. Philidelphia: Elsevier; 2012. p. 74-95.

20. Holihan JL, Nguyen DH, Nguyen MT, Mo J, Kao LS, et al. Mesh location in open ventral hernia repair: a systematic review and network meta-analysis. World J Surg 2016;40:89-99.

21. Novitsky YW, Elliott HL, Orenstein SB, Rosen MJ. Transversus abdominis muscle release: a novel approach to posterior component separation during complex abdominal wall reconstruction. Am J Surg 2012;204:709-16.

22. Harth KC, Blatnik JA, Rosen MJ. Optimum repair for massive ventral hernias in the morbidly obese patientis panniculectomy helpful? Am J Surg 2011;201:396-400.

23. Saulis AS, Dumanian GA. Periumbilical rectus abdominis perforator preservation significantly reduces superficial wound complications in \&quot;separation of parts\&quot; hernia repairs. Plast Reconstr Surg 2002;109:2275-80.

24. Maloney SR, Schlosser KA, Prasad T, Kasten KR, Gersin KS, et al. Twelve years of component separation technique in abdominal wall reconstruction. Surgery 2019;166:435-44.

25. Colavita PD, Wormer BA, Belyansky I, Lincourt A, Getz SB, et al. Intraoperative indocyanine green fluorescence angiography to predict wound complications in complex ventral hernia repair. Hernia 2016;20:139-49.

26. Carbonell AM. Interparietal hernias after open retromuscular hernia repair. Hernia 2008;12:663-6.

27. Davis JR, Villarreal JE, Cobb WS, Carbonell AM, Warren JA. Interparietal hernia complicating retromuscular ventral hernia repair. Am Surg 2016;82:658-9.

28. Garcia-Ruano A, Deleyto E, Garcia-Fernandez S. VAC-instillation therapy in abdominal mesh exposure: a novel indication. J Surg Res 2016;206:292-7.

29. Weyhe D, Cobb W, Lecuivre J, Alves A, Ladet S, et al. Large pore size and controlled mesh elongation are relevant predictors for mesh integration quality and low shrinkage - systematic analysis of key parameters of meshes in a novel minipig hernia model. Int $\mathrm{J}$ Surg 
2015;22:46-53.

30. Gray SH, Vick CC, Graham LA, Finan KR, Neumayer LA, et al. Risk of complications from enterotomy or unplanned bowel resection during elective hernia repair. Arch Surg 2008;143:582.

31. Yaghoobi Notash A, Yaghoobi Notash A, Seied Farshi J, Ahmadi Amoli H, Salimi J, et al. Outcomes of the rives-stoppa technique in incisional hernia repair: ten years of experience. Hernia 2007;11:25-9.

32. Brandi CD, Roche S, Bertone S, Fratantoni ME. No enterocutaneous fistula development in a cohort of 695 patients after incisional hernia repair using intraperitoneal uncoated polyproylene mesh. Hernia 2017;21:101-6.

33. Quinn M, Falconer S, McKee RF. Management of enterocutaneous fistula: outcomes in 276 patients. World J Surg 2017;41:2502-11.

34. Schecter WP. Management of enterocutaneous fistulas. Surg Clin North Am 2011;91:481-91.

35. Kao AM, Arnold MR, Otero J, Huang LC, Prasad T, et al. Comparison of outcomes after partial versus complete mesh excision. Ann Surg 2019; Epub ahead of print doi: 10.1097/sla.0000000000003198

36. Kokotovic D, Bisgaard T, Helgstrand F. Long-term recurrence and complications associated with elective incisional hernia repair. J Am Med Assoc 2016;316:1575-82.

37. Warren JA, McGrath SP, Hale AL, Ewing JA, Carbonell AM, et al. Patterns of recurrence and mechanisms of failure after open ventral hernia repair with mesh. Am Surg 2017;83:1275-82.

38. Cobb WS, Kercher KW, Heniford BT. The argument for lightweight polypropylene mesh in hernia repair. Surg Innov 2005;12:63-9.

39. Petro CC, Nahabet EH, Criss CN, Orenstein SB, von Recum HA, et al. Central failures of lightweight monofilament polyester mesh causing hernia recurrence: a cautionary note. Hernia 2015;19:155-9.

40. Deerenberg EB, Harlaar JJ, Steyerberg EW, Lont HE, van Doorn HC, et al. Small bites versus large bites for closure of abdominal midline incisions (STITCH): a double-blind, multicentre, randomised controlled trial. Lancet 2015;386:1254-60.

41. Menzo E Lo, Hinojosa M, Carbonell A, Krpata D, Carter J, et al. American Society for Metabolic and Bariatric Surgery and American Hernia Society consensus guideline on bariatric surgery and hernia surgery. Surg Obes Relat Dis 2018;14:1221-32. 